\title{
ФІЗІОЛОГІЧНІ ЕФЕКТИ СВІТЛА З ПЕРЕТВОРЕНОЮ ФУЛЛЕРЕНОМ ПРОСТОРОВОЮ СТРУКТУРОЮ
}

\author{
С. О. Ґуляр ${ }^{1,4}$, 3. А. Тамарова ${ }^{1}$, Є. К. Кириленко ${ }^{3}$, \\ Н. Б. Філімонова ${ }^{2}$, М. Ю. Макарчук², Ю. М. Кривдюк ${ }^{2}$ \\ ${ }^{1}$ /нститут фрізіології ім. О. О. Богомольця НАН України \\ ${ }^{2}$ Київський національний університет імені Тараса Шевченка \\ ${ }^{3}$ Науково-технічний центр «BIPIA-Ltd» \\ ${ }^{4}$ Інтернаціональний медичний інноваційний центр Цептер
}

\begin{abstract}
Експериментально показано, що перетворене фруллереном поляризоване (апарат Біоптрон) або розсіяне (окуляри Tesla HyperLight Eyewear) світло викликають достовірні фрізіологічні зміни, виявлені в антиноцицептивній сфрері, ЦНС і ВНД. Виявлено достовірне ослаблення больової реакції (анальгетичну дію) і збільшення тривалості сну (заспокійливу дію). Багатомісячне перебування тварин під фуллереновим освітленням виявило уповільнення розвитку деяких ознак старіння. Мозаїка ЕЕГ-активності свідчила про розвиток більш споглядального ставлення до інтроспективного емоційних переживань минулих подій при активному залученні їх в образне моделювання гіпотетичного майбутнього. Встановлено, що в природних умовах перетворене фулереном світло полегшує виконання зоровомоторних тестів. При тривалому коректурному навантаженні фуллеренове світло, сприяло збільшенню швидкості перероблення інфрормації в зоровому аналізаторі, підвищувало уважність і послаблювало стомлюваність. Якість виконання інтенсивного розумового навантаження зростала. Моделювання «засліплення» водія за даними сенсомоторних реакцій на тлі ЕЕГ мозку людини виявило збільшення швидкості міжпівкульних інформаційних процесів і підвищення якості та ефективності прийнятих рішень.
\end{abstract}

Ключові слова: поляризоване світло, Біоптрон, суллерен, біль, анальгезія, сон, десролтні мережі мозку, розумова працездатність, вища нервова діяльність.

\section{PHYSIOLOGICAL EFFECTS OF LIGHT WITH SPATIAL STRUCTURE CONVERTING BY FULLERENE}

\author{
S. O. Gulyar ${ }^{1,4}$, Z. A. Tamarova ${ }^{1}$, Ye. K. Kyrylenko ${ }^{3}$, \\ N. B. Filimonova ${ }^{2}$, N. Yu. Makarchuk², Yu. N. Kryvdiuk ${ }^{2}$ \\ ${ }^{1}$ Bogomoletz Institute of Physiology, National Academy of Sciences of Ukraine \\ ${ }^{2}$ Taras Shevchenko National University of Kyiv \\ ${ }^{3}$ Scientific and Technical Centre «VIRIA-Ltd» \\ ${ }^{4}$ Zepter International Medical Innovation Center
} passing through it at the nanoscale caused interest in determining its physiological action. Experimental studies included assessment of pain and non-pain behavioral reactions of animals (formalin test). Electroencephalography was used to determine the characteristics of the response of the brain's default systems and human sensor-motor functions. The state of the higher nervous activity reflected the dynamics of indicators of mental performance during visual loads of varying complexity. We used polarized light (Bioptron device with a fullerene filter) and diffused light (Tesla HyperLight Eyewear glasses). It has been experimentally shown that light converted by fullerene causes significant physiological changes detected in the antinociceptive sphere, the central nervous system (CNS) and higher nervous activity (HNA). It was revealed a significant weakening of the pain response (analgesic effect) and an increase in the duration of sleep (sedative effect) with the application of «carbon» light on the locus of pain or on the acupuncture point. The many-month stay of animals under natural light passed through the fullerene filter revealed a slowdown in the development of some signs of aging (according to the dynamics of body weight, motor activity) in the absence of negative behavioral changes.

Materials and methods. Results. The EEG-activity mosaic testified to the development of a more contemplative attitude to the introspective emotional experiences of past events with their active involvement in the figurative modeling of a hypothetical future. It has been established that in natural conditions the light transformed by fullerene facilitates the performance of the visual-motor task in the black-and-white digital sorting test by $16.5 \%$. This was equivalent to reducing the age of the CNS structures that participated in the processing of digital information by $14.3 \%$. The quality of work related to switching attention was improved: according to the color digital test, the execution time was shortened to $105.6 \mathrm{~s}$ versus $118.0 \mathrm{~s}$ in the control, the average time for switching attention from one character to another was $-1.85 \mathrm{~s}$, against 2.03 $\mathrm{s}$, and errors were made 2.7 times less. With prolonged correctional load, fullerene light contributed to the increase of the information processing rate in the visual analyzer, it increased attentiveness and weakened fatigue. The concentration ratio

(c) С. О. Гуляр, 3. А. Тамарова, Є. К. Кириленко, Н. Б. Філімонова, М. Ю. Макарчук, Ю. М. Кривдюк 
at the end of the performance of a long proof test in fullerene glasses decreased less (by $16.2 \%)$ than in intact (23.6 \%) or placebo $(28.5 \%)$ conditions. The latent period of the reaction to the appearance of the symbol was lower, and its deviation was less when compared with comparison groups. Switching attention with a load of 2000 characters has always been faster: $0.537 \pm 0.05 \mathrm{~s}$ (fullerene), $0.572 \pm 0.06 \mathrm{~s}$ (intact) and $0.544 \pm 0.07 \mathrm{~s}$ (placebo). Similar dynamics existed for the latent period of the reaction, its physiological duration, and the time taken to complete the attention-shifting test. The quality of performance of intense mental load increased: the number of errors was 39.97 points, whereas, in intact conditions 49.83, and placebo - 46.11 points.

Conclusions. Modeling the standard situation in which the driver of a vehicle gets under extreme illumination, according to the dynamics of sensorimotor reactions and their correlation against the background of the EEG of the human brain, revealed an increase in the rate of inter-hemispheric information processes and an increase in the quality and effectiveness of decisions made.

Key words: polarized light, Bioptron, fullerene, pain, analgesia, sleep, default brain networks, mental performance, higher nervous activity.

\title{
ФИЗИОЛОГИЧЕСКИЕ ЭФФЕКТЫ СВЕТА С ПРЕОБРАЗОВАННОЙ ФУЛЛЕРЕНОМ ПРОСТРАНСТВЕННОЙ СТРУКТУРОЙ
}

\author{
С. А. Ґуляр ${ }^{1,4}$, 3. А. Тамарова ${ }^{1}$, Е. К. Кириленко ${ }^{3}$, \\ Н. Б. Филимонова ${ }^{2}$ Н. Е. Макарчук², Ю. Н. Кривдюк ${ }^{2}$ \\ ${ }^{1}$ Институт фризиологии им. А. А. Богомольца НАН Украины \\ ${ }^{2}$ Киевский национальный университет имени Тараса Шевченко \\ ${ }^{3}$ Научно-технический чентр «ВИРИА-Ltd» \\ ${ }^{4}$ Интернациональный медицинский инновационный центр Цептер
}

\begin{abstract}
Экспериментально показано, что преобразованный фуллереном поляризованный (аппарат Биоптрон) или рассеянный (очки Tesla HyperLight Eyewear) свет вызывают достоверные фризиологические изменения, выявленные в антиноцицептивной сорере, ЦНС и ВНД. Выявлено достоверное ослабление болевой реакции (анальгетическое действие) и увеличение продолжительности сна (успокаивающее действие). Многомесячное пребывание животных под фруллереновым освещением выявило замедление развития некоторых признаков старения. Мозаика ЭЭГактивности свидетельствовала о развитии более созерцательного отношения к интроспективным эмоциональным переживаниям прошлых событий при активном вовлечении их в образное моделирование гипотетического будущего. Установлено, что в естественных условиях преобразованный фруллереном свет облегчает выполнение зрительно-моторных тестов. При длительной корректурной нагрузке фууллереновый свет, способствовал увеличению скорости переработки иноормации в зрительном анализаторе, повышал внимание и ослаблял утомляемость. Качество выполнения интенсивной умственной нагрузки возрастало. Моделирование «ослепления» водителя по данным сенсомоторных реакций на фоне ЭЭГ мозга человека выявило увеличение скорости межполушарных информационных процессов и повышение качества и эффрективности принимаемых решений.
\end{abstract}

Ключевые слова: поляризованный свет, Биоптрон, фуллерен, боль, анальгезия, сон, дефолтные сети мозга, умственная работоспособность, высшая нервная деятельность. 
Введение. В последние годы появились новые материалы, компоненты которых, имея наноразмеры, способны изменять свойства проходящего через них света. Это обусловило интерес к определению его физиологического действия. В конце XX века открыты новые аллотропные формы углерода: фуллерен [1] и графен [2], свойства которых создают неизвестные ранее технологические возможности. Молекула фуллерена (рис. 1, А) имеет форму сферического многогранника (усеченного икосаэдра). Атомы углерода в количестве от 20 до 200 объединяются в молекулы, наиболее распространенной из них является форма $\mathrm{C}_{60}$. Графен представляет собой плоскую сетевую структуру (рис. 1, Б), состоящую из 1-2 слоев соединенных между собой атомов углерода. Одним из свойств материалов, содержащих фуллерен или графен, является способность влиять на световой поток. Соответственно такой свет может приобретать дополнительные свойства [3-5]. В настоящей статье мы приводим результаты, полученные при применении фуллеренового фильтра в разных вариациях.

Существует гипотеза, основанная на физическом описании процесса преобразования входящего света молекулой $\mathrm{C}_{60}$ [6], о возможности использования такого пути для изменения конфигурации квантово-волновой структуры светового потока [7-8]. Предварительная подготовка света состоит в его поляризации. Она достигается путем отражения рассеянного света под углом Брюстера от многослойного стекла-зеркала (аппарат Биоптрон). Полученный таким путем полихроматический (галогенный) вертикально линейно поляризованный (ПАЙЛЕР) свет пропускается через слой органического стекла (полиметил-мета-акрилат, ПММА), в которое внесены и равномерно распределены при помощи ультразвука наноструктуры (молекулы) фуллерена. В результате фотоны света дополнительно модулируются угловым импульсом (эффект Фарадея [9]), вследствие чего происходит их отклонение в электромагнитных полях. Вертикально линейно поляризованный свет последовательно переходит в горизонтально линейно поляризованный свет по порядку, определяемому законом Фибоначчи и далее закручивается в спирально-кольцевую структуру (тороид Теслы). В результате распределение квантов в световом потоке по форме приближается к расположению семян в шляпке подсолнечника (рис. 2). Такой световой поток назван гиперполяризованным (синонимы: гиперполяризованный свет с квантовыми наносвойствами, квантовый гиперсвет, «углеродный» и т. п.), а сами фильтры — фуллереновыми, нанофотонными, углеродными и другими.

В соответствии с гипотезой Коруги [7], преобразование пространственной структуры света происходит потому, что 20 шестиугольников (парамагниты) оболочки молекулы $\mathrm{C}_{60}$ вращательно рассеивают проникшие внутрь поляризованные (колеблющиеся в одной плоскости) фотоны, а 12 пятиугольников (диамагниты) той же оболочки дополнительно их поворачивают, последовательно распределяя их в потоке по закону Фибоначчи [8]. Часть фотонов света проходит через молекулу $\mathrm{C}_{60}$ без изменения направления ( 12 \%), вторая их часть взаимодействует с энергиями полных угловых моментов, упорядоченных по закону Фибоначчи Ф (54 \%), а третья - ( 28\%) преобразуется и упорядочивается по закону Фибоначчи $\varphi$ [8]. Изменения траектории фотонов обусловлены тем, что атомы углерода, входящие в молекулу $\mathrm{C}_{60}$, могут влиять на эти траектории собственными электромагнитными свойствами, подчиняющимися влиянию икосаэдрической симметрии оболочки.

Вращающаяся со скоростью $1.8 \times 10^{10} \mathrm{c}^{-1}$ пространственная сеть атомов углерода, входящая в молекулу $\mathrm{C}_{60}$, в соответствии с законом распределения Фибоначчи-Тесла $\left(\Phi^{2}+\varphi^{2}=3\right)$ дополнительно перераспределяет световой электромагнитный поток. (Это перераспределение описывается числовой последовательностью, в которой каждый последующий член ряда равен сумме двух предыдущих, то есть: 1, 1, 2, 3, 5, 8, 13, 21, 34, 55, 89, 144, 233, 377, 610, 987 ... В результате по мере увеличения чисел отношение двух соседних членов этого ряда асимптотически приближается к точной пропорции Золотого сечения, $1: 1,618)$.

Когда свет проходит через фуллереновый материал толщиной 2 мм (106 слоев молекул $\mathrm{C}_{60}$ - каждая с 20 гексагонами и 12 пентагонами [7]), происходит 1018 изменений движения квантов, создается идеальная рандомизация фотонов с вероятностью $1 \mathrm{~K}$ созданию формы «подсолнечника» (1 / 0.61803 ... $=1.61803$...). С учетом вращения молекулы $\mathrm{C}_{60}$ в итоге создается новая более упорядоченная (симметричная) спиральная конфигурация потока. Она становится сопоставимой с симметрией встречного потока квантов биомолекул, имеющих закономерности строения, совпадающие с распределением Фибоначчи-Тесла и правилом «Структура света соответствуют структуре вещества» [10]. Это создает зеркальный резонансный эффект с возможностями для более эффективного использования (поглощение) 
каждого кванта и запуска вторичных ответных реакций на молекулярном уровне.

Дополнительно в фуллереновых стеклах происходит спектральное изменение прошедшего света за счет поглощения. Результаты преобразования фуллереном спектра видимого света показаны на рис. 3. Заметно ослабление высокоэнергетической части солнечного спектра, т. е. плотности мощности в УФ, фиолетовом, синем, зеленом и желтом диапазонах.

Свет различных поляризованных волновых диапазонов создает неодинаковый биологический эффект, о чем имеются экспериментальные доказательства [11-12]. Что касается светового потока «углеродного» происхождения, в первую очередь было целесообразно определить наличие его действия на тканевом и системном уровнях. Поскольку поли- и монохроматический поляризованный свет уже применяется с профилактическими и лечебными целями [13], то представило дополнительный интерес выявление клинических возможностей света, преобразованного фуллереном. При положительном ответе возникает перспектива его применения с профилактической или лечебной целью.

Цель работы: ознакомить с результатами экспериментальных исследований о физиологических эффектах света. Показать, что преобразованный фуллереном поляризованный или рассеянный свет вызывают достоверные физиологические изменения, выявленные в антиноцицептивной сфере, ЦНС и ВНД.

Материал и методы исследования. Проведены мультицентровые исследования с единой аппаратурно-технической базой и методологическим подходом. Обязательным было применение контрольных и плацебо исследований. Источниками света являлись аппараты Биоптрон-компакт и БиоптронПро-1, в ряде серий - дневной рассеянный свет. Фуллереновые фильтры применялись в трех вариантах: в сборе с аппаратом Биоптрон, в виде очков, оснащенных указанными фильтрами в оправе (Tesla HyperLight Eyewear, THE) и в виде фуллеренового покрытия клетки для животных. Эксперименты разных серий проводились на животных и с участием человека.

Исследование возможности влияния фуллеренового света на болевой синдром выполнено на животных (белые мыши) в однотипных экспериментальных условиях сериями по 1015 особей [14]. Очаг болевого воспаления создавали подкожным введением 30 мкл 5 \%-го раствора формалина (в 0,9 \% растворе $\mathrm{NaCl}$ ) в тыльную поверхность стопы (формалиновый тест).
Об интенсивности боли судили по продолжительности реакции лизания очага боли за последовательные 10-ти минутные интервалы времени после световой аппликации (на очаг воспаления или точку акупунктуры Е-36) и за 60 минут наблюдения. Регистрировали также неболевые поведенческие реакции (сон, умывание, бег и еда).

Эффект длительного (8-ми месячного) влияния фуллеренового света на продолжительность и качество жизни изучался на стареющих лабораторных животных (белые мыши-самцы в возрасте 1 года). Мыши получали дневной свет, пропущенный через фуллереновый фильтр (клетки были накрыты пластинами из фуллеренового материала). Контролем служила группа аналогичных животных, находившаяся под естественным светом. Регистрировали изменения массы тела, общую двигательную активность (тест «открытое поле») и суммарную продолжительность жизни животных в каждой группе.

Для оценки умственной работоспособности человека [15-18] была разработана новая методическая база, основанная на клавиатурно-дисплейных технологиях. Специально разработаны тесты, обладающие различной чувствительностью по отношению к устойчивым («старым») и более подвижным «молодым» нервным связям. Особенностью этих тестов является полная объективизация оценки результатов тестирования и исключение стандартных технических помех. Новой является возможность определения и учета скорости тактильной реакции испытуемого с точностью до 1 мс. Кроме того, этот показатель используется для определения истинной функциональной скорости процессов в ЦНС человека. Учитываются также технические возможности процессора конкретного компьютера, на котором выполняется тест. Согласно циклограмме тестирования каждый испытуемый выполнял корректурные тесты в первый день без очков, на следующий день - в фуллереновых очках THLEW и на третий день — в очках-плацебо.

Тест монохроматической цифровой сортировки, выявляет сообразительность, адекватность пациента и его умственную работоспособность при выполнении упрощенных задач. Тест основан на обработке монохроматических таблиц ГорбоваШульте (поиск неупорядоченных цифр 1-25 с максимальной скоростью). В результате выполнения теста фиксируется время выполнения задания с учетом тактильной реакции, а также вычисляется функциональный возраст структур, отвечающих за выполнение этой зрительно-моторной задачи. 
Тест «Черно-красная таблица» (Горбова-Шульте) [19-20] в нашей модификации (рис. 4) используется для оценки скорости переключения внимания в условиях, требующих повышенной концентрации и быстрой реакции.

Испытуемому предлагается отметить максимально быстро черные цифры от 1 до 25, а красные - от 24 до 1. После фиксации последней цифры автоматически учитывается длительность работы, число и время возникновения ошибок, как в выборе черных чисел, так и красных, сбоев (потеря последовательности). При этом имеется возможность регистрации точного времени каждого выбора, а также расчета интервала между каждым выбором (затраты времени на принятие решения). Для унификации оценки интервальных характеристик теста разработана модифицированная нами бальная оценка (максимальное количество балов 250).

Тест корректурный простой, предназначен для количественного определения физиологических возможностей человека к переработке зрительной информации в условиях дефицита времени (рис. 5).

Таблица представляет собой 1000 и более зрительных образов (колец Ландольта, каждое из которых содержит одинаковое количество информации), ориентированных в случайном порядке по 8 направлениям. Задача состоит в вычеркивании (отметке) заданного кольца с максимальной скоростью. При повторной работе с таблицей расположение колец меняется случайным образом. Учитывается время фиксации внимания человека на кольцах с разной ориентацией разрыва.

Погрешность определения времени для процесcopa Intel Core i3 составляет 0,001 с. Дополнительно в тесте введена возможность определения времени реакции человека на появление символа. Такой учет определения времени тактильной реакции человека дает возможность определить чистую физиологическую длительность обработки одного символа. Расчет общего количества переработанной информации проводится по формулам Ландольта [17], Генкина, Медведева, Шека [21] с учетом информационной емкости символов, по эмпирической формуле с учетом граничных условий и экспертизы динамики прохождения теста, а также по специально разработанным формулам оценки механизмов изменения интегральных показателей. Автоматически подсчитывалось количество правильно и неправильно обработанной информации, забывчивость и сбои, сосредоточенность, скорость обработки информации и каждого правильно обработанного символа, время переключения и фиксации внимания на одном кольце, общее количество переработанной информации, скорость обработки задания, среднее время, затраченное на обработку 1 бита информации.

Тест корректурный избирательный (с переключением внимания и оценкой долговременной памяти): на фоне задания выполнять простой корректурный тест, когда условие теста показывается обследуемому на короткое время (2-5 с), после чего он должен держать это условие в памяти. Таких смен на протяжении теста происходит несколько. Такой тест позволяет выявить устойчивость наиболее «молодых» нервных связей и тенденции к ослаблению умственной работоспособности (на основании способности сохранять внимание и переключаться на выполнения нового кода задания). Результаты обрабатываются по описанной выше схеме с учетом новых данных о сбоях при выполнении указанного условия и бальной оценке.

Тест корректурной нагрузки с переключением внимания, имитирующий интенсивную работу в социальных сетях или интенсивную офисную (компьютер) деятельность. Предусмотрено предъявление испытуемому корректурной таблицы, содержащей увеличенное количество колец Ландольта (2000-10000 и выше), требующей длительного времени обработки. Оцениваются показатели по приведенной выше методике.

В конце выполнения каждого из тестов автоматически создавался текстовый протокол, в котором фиксировались показатели, полученные в ходе тестирования, а также в виде опции - накопление результатов каждого обследования в таблице для последующей вторичной или статистической обработки, графического представления динамики прохождения теста (рис. 6).

Для регистрации и анализа ЭЭГ человека использовали комплекс Нейрон-Спектр-4/ВП (НейроСофт). Запись проводили при дневном свете умеренной интенсивности монополярно, с частотой квантования 500 Гц, режекторным фильтром 50 Гц, верхнем и нижнем фильтрами частот соответственно 0,5 и 200 Гц, референтные электроды располагались на мочках ушей. Использовали мостиковые посеребренные электроды, которые накладывались согласно международной системе 10-20 в 19 отведениях Fp1, Fp2, F3, F4, F7, F8, Fz, C3, C4, Cz, T3, T4, T5, T6, P3, P4, Pz, O1, O2. В каждом отведении для частотных диапазонов ЭЭГ — Delta (0,5-3,9 Гц), Theta (4,0-7,9 Гц), Alpha 
(8,0-12,9 Гц), Beta1 (13,0-19,9 Гц) и Beta2 (20,035,0 Гц) в программе Нейрон-Спектр рассчитывалась полная мощность спектра. Обследуемые пребывали в состоянии спокойного бодрствования с открытыми глазами в течение 5 мин. При обследовании в первый день применялись очки с фуллереновым фильтром, а в другой день - очки аналогичного спектра без фуллерена (плацебо).

При проведении теста с простой сенсомоторной реакцией, совмещенной с ЭЭГ, обследуемому на экране компьютера давалась инструкция, следуя которой на появление изображения (квадрата) он должен был как можно быстрее ответить нажатием клавиши. После адаптации последовательно следовали еще 100 изображений основной группы. Пауза между ними выбиралась случайно из интервала 500-600 мс для того, чтобы следующий сигнал был ожидаемым, но не было настройки на ритм предъявления изображений. Регистрировали латентный период (ЛП) простой сенсомоторной реакции с точностью до 10 мс. Вычисляли скорость простой сенсомоторной реакции (Simple Response Time, SRT, мc) как среднее значение из 100 реакций. В разных сериях обследований предъявляли ярко-голубой квадрат на темном фоне (контроль); белый квадрат на ярко-голубом фоне (яркий солнечный день), в плацебо-очках и в очках с фуллереновым фильтром.

В тесте по определению времени реакции выбора, совмещенном с регистрацией ЭЭГ, была использована компьютерная модификация классической методики при предъявлении обследуемому серии из 200 стимулов. При этом в случайном порядке и с неодинаковыми паузами на экране компьютера появлялся квадрат или треугольник, на которые надо было реагировать соответственно правой или левой рукой. На ошибочную реакцию компьютер не реагировал, а ожидал правильной реакции, то есть регистрировалось время, затраченное на правильную идентификацию изображения (с точностью до 10 мс).

Для статистического анализа использовалась программа Statistica. Критический уровень значимости при проверке статистических гипотез принимали равным 0,05. Распределение переменных проверяли на нормальность критерием ШапироУилка. Поскольку распределение большинства переменных отличалось от нормального $(\mathrm{p} \leq 0,5)$, сравнение двух зависимых выборок проводили критерием Уилкоксона, а данные на графиках представлялись в виде Ме (25 \%, 75 \%) [22].
Результаты и их обсуждение. Результаты исследований позволили получить начальное впечатление о новом световом факторе, которое может иметь клиническое значение. Биологическая эффективность ПАЙЛЕР-света, прошедшего через нанослои углерода (фуллерен) была доказана при помощи экспериментальной модели боли, как одного из распространенных синдромов. При аппликациях «углеродного» света на очаг боли/ воспаления или на точку акупунктуры (рис. 7) было выявлено ослабление болевой реакции (анальгетическое действие) и увеличение продолжительности сна (рис. 8, успокаивающее действие) [23-24].

Экспериментально было определено место фуллеренового света (по эффективности действия) в полной световой палитре [24, 25]. Анальгетическая рейтинговая позиция «углеродного» света определялась на основании статистического сравнения интенсивности биологических реакций с полученными по единой методике результатами действия других поли- и монохроматических (оранжевый, желтый, зеленый, голубой, синий и фиолетовый) диапазонов поляризованного света.

При аппликации ПАЙЛЕР-света, прошедшего через фуллереновый фильтр, анальгезия составляла 38,5 \% (точка акупунктуры Е-36) и 43,5 \% (очаг боли). По эффективности подавления боли фуллереновый фильтр занимал среднее место между другими фильтрами (рис. 9).

Вторым наиболее заметным проявлением биологической эффективности ПАЙЛЕР-света, прошедшего через фуллереновый фильтр, является его мощное успокаивающее действие. Критерием является изменение продолжительности сна у животных с очагом воспалительной боли. При аппликации на ТА Е-36 из всех изученных диапазонов света фуллереновый свет оказывал наибольшее успокаивающее влияние (рис. 10). Длительность сна возрастало в 3,2 раза по сравнению с контрольной группой, тогда как другие диапазоны удлиняли сон только в 1,6-2,4 раза. При действии непосредственно на очаг боли свет, прошедший через фуллереновый фильтр, удлинял сон в 2 раза (другие фильтры — в 1,4-2,3 раза).

При анализе эффектов света, модифицированного молекулярным углеродом, возникает вопрос, насколько это явление уникально. Использование единой экспериментальной технологии применительно к болевому синдрому позволило сопоставить полученные результаты с данными для основных монохроматических диапазонов света. Первым итогом стало понимание того, что по противоболевому 
действию фуллереновый свет входит в группу наиболее эффективных диапазонов видимого света, дающих достоверный анальгетический эффект. Вторым, и весьма значимым, является факт седативности, который ранее ни в одном из исследованных монохроматических вариантов света не был настолько выраженным. Важно, что эти результаты подтверждают возможность как локального, так и удаленного (через точку акупунктуры) эффектов, получаемых бесконтактным и нефармакологическим путем. Все это свидетельствует о том, что мы имеем дело с фактором, который может дать дополнительные возможности, важные для практической медицины.

Получение вышеописанных данных поставило вопрос о возможности возникновения биологического эффекта от рассеянного света, прошедшего через фуллереновый фильтр. В этом случае световая аппликация происходит не только на дермальном, но и окулярном уровнях. Поскольку такой свет является низкоинтенсивным, то была применена методика длительного пребывания животного под естественным дневным светом, проходящим через фуллереновый фильтр.

Длительный 8-ми месячный эксперимент [26] проводился на мьшах-самцах в возрасте 1 года («старые» животные). Контролем служила группа аналогичных животных, находившаяся под естественным светом. Установлено, что после 9 недель наблюдения масса тела у животных контрольной группы начинала постепенно снижаться (один из признаков старения). В то же время средняя масса тела у животных экспериментальной группы оставалась на прежнем уровне или даже возрастала (рис. 11). Максимальное различие между двумя группами стало заметным через 32 недели. Прирост массы тела животных, находившихся под фуллереновым светом, составлял 20,5 \%, а в контрольной группе он снижался до 13,8\%). Тест, характеризующий свободную подвижность («открытое поле»), показал, что по мере продолжающегося естественного старения животных двигательная активность у них снижалась. Однако в контрольной группе этот процесс был быстрее. Доказано, что «фуллереновый» свет не нарушал естественного поведения животных (сон, пищевое поведение и другое). Все это свидетельствовало об улучшении качества жизни «фуллереновых» животных: позитивная динамика массы тела, сохранение двигательной активности, отсутствие негативных поведенческих изменений. Достоверного влияния «фуллеренового» света на продолжительность жизни животных не выявлено. Однако при этом длительность жизни отдельных особей существенно превысила средние величины (до 14 мес). С учетом этих данных животные экспериментальной группы прожили суммарно 1452 дня, а контрольные животные - 1036 дней.

Таким образом, впервые было показано, что длительное до 8 месяцев освещение тела животного светом, прошедшим через нанофотонный фуллереновый фильтр, не оказывает отрицательного влияния на физиологические и функциональные показатели, а некоторые из них даже улучшает. Замедляется развитие некоторых признаков старения: потеря массы тела и уменьшение подвижности. В данном случае реализация эффектов наномодифицированного светового излучения достигалась одновременно двумя путями - трансдермальным и окулярным.

Выделение роли окулярной световой аппликации в качестве основного пути транспорта световых фотонов позволило глубже понять механизмы физиологических ответов. Для этой цели была изучена активность крупномасштабных молчащих (дефолтных) сетей мозга человека M. Raichle [27] Окулярный путь светового воздействия обеспечивался применением очков ТНЕ, для плацебо применялись очки в аналогичной оправе с фильтром, подобным к THE по световому диапазону, но не содержащим фуллерена, контроль — очки с прозрачными стеклами. Результаты оценивались на основании анализа электрической активности головного мозга человека (ЭЭГ) [28-30].

Были получены достоверные отличия в дельта- и тета-диапазонах в пробе «Закрытые глаза», которые указывали на изменения состояния дефолтных сетей. Сниженная ЭЭГ-активность в альфа-диапазоне в правой фронтальной зоне свидетельствовала о повышении межрегиональной синхронизации с вовлечением образной информации в процессы внутреннего мышления (рис. 12). Повышенная ЭЭГактивность в дельта-диапазоне в височной зоне свидетельствовала об активации процессов образного моделирования будущего, а сниженная активность ЭЭГ в задней височной зоне - об уменьшении внимания к внутренним процессам, т. е. о расслаблении. Повышенная ЭЭГ-активность в тета-диапазоне в задней сингулярной коре свидетельствовала об усилении процессов интроспективного эмоционального переживания прошлых событий.

Активация головного мозга в тета-диапазоне происходит при кодировании (запоминании) информации и ее декодировании (воспоминаниях) [31]. Активность в этом диапазоне связывают с организацией нейросети под задачу и последующим контролем за ошибочными реакциями [32]. 


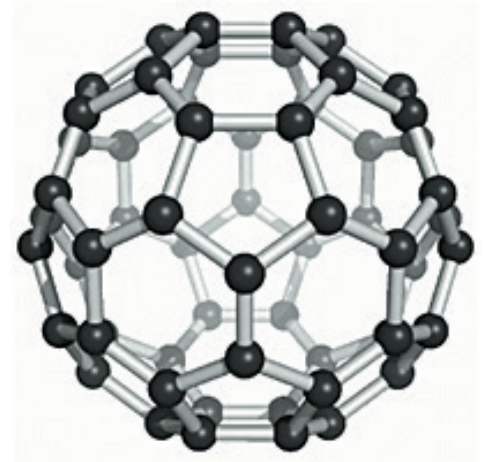

A

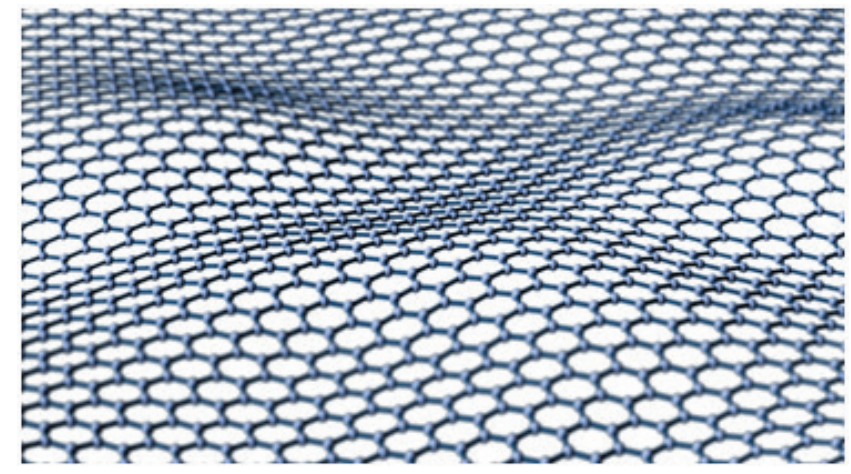

Б

Рис. 1. Схемы структуры молекул фуллерена (А) и графена (Б)

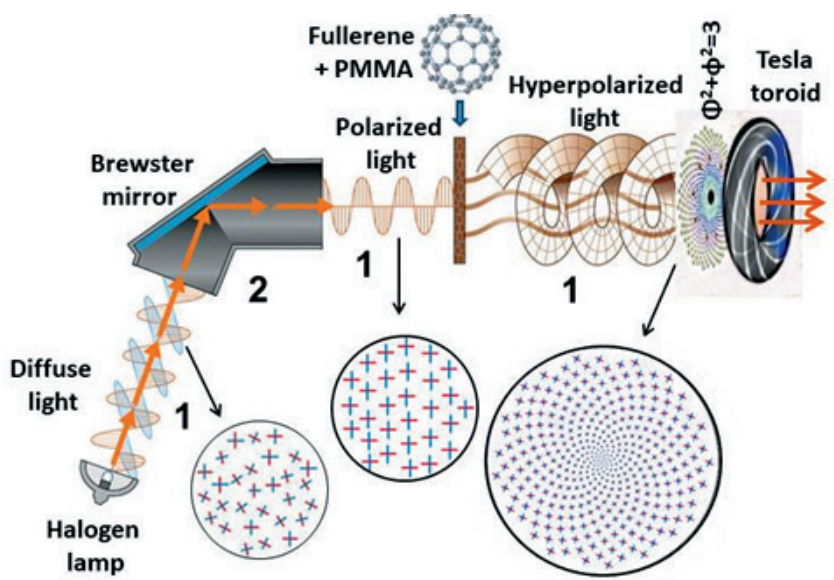

Рис. 2. Схема преобразования рассеянного света в линейно поляризованный и гиперполяризованный (в соответствии с гипотезой Коруги [7]): 1 — схема векторов колебаний световых электромагнитных волн, 2 — поляризатор Брюстера аппарата Биоптрон; различия в векторной структуре световых потоков показаны отдельно (обведены кругами)
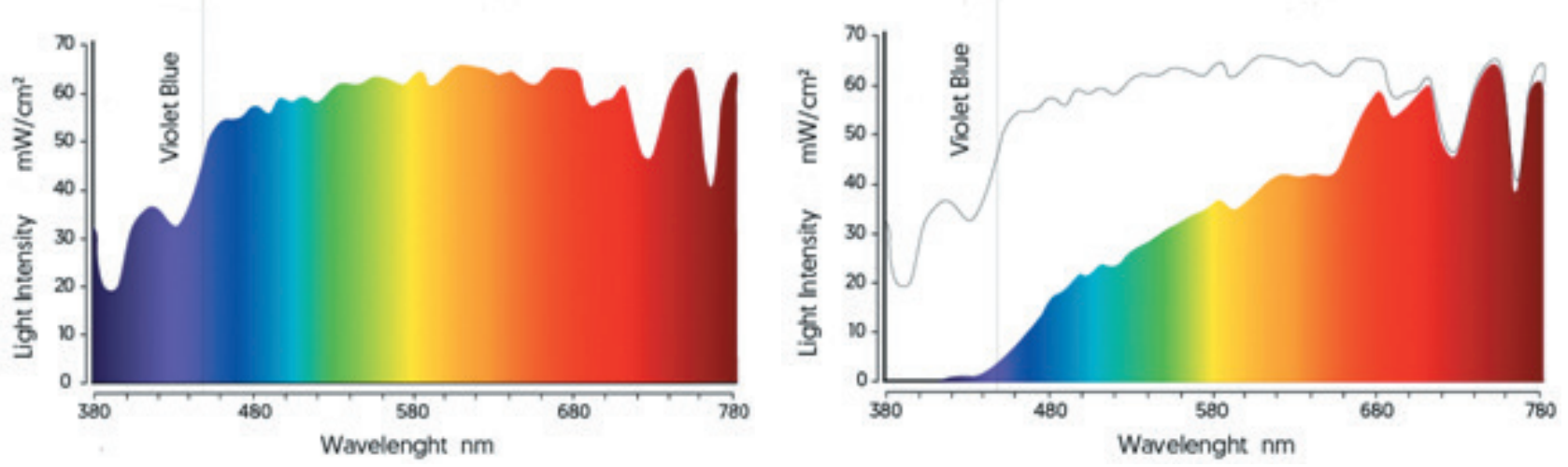

Рис. 3. Натуральные и преобразованные фуллерен+ПММА-материалом спектры солнечного света 


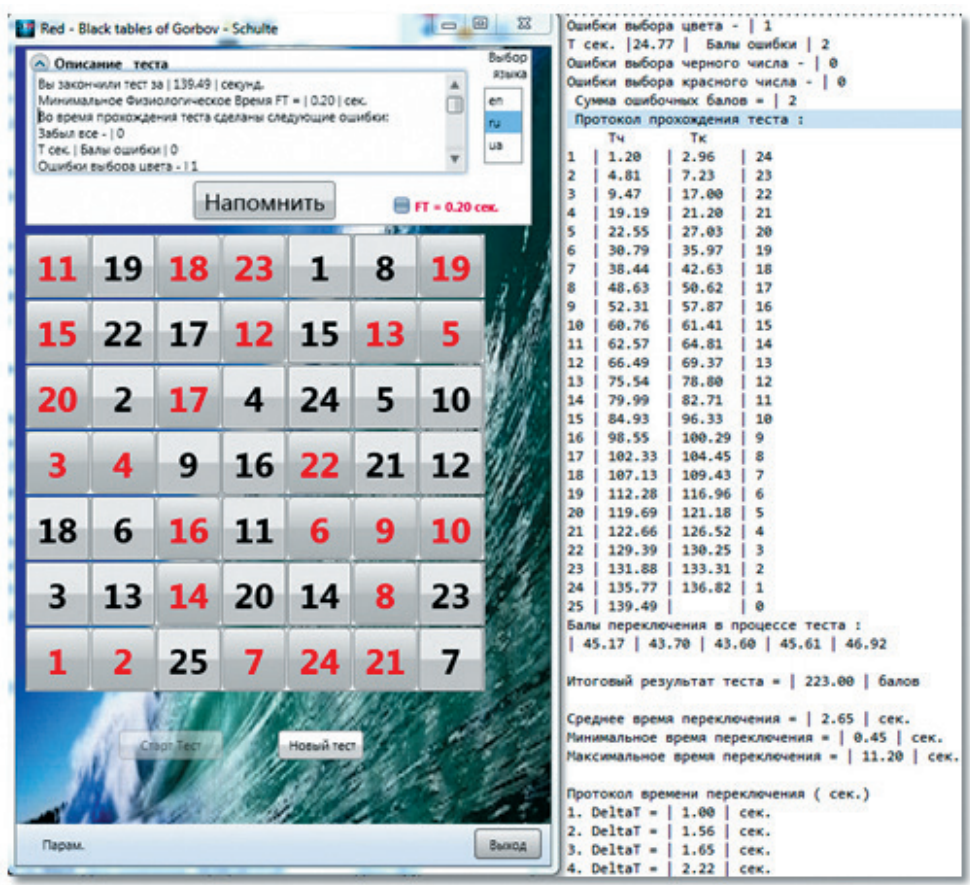

Рис. 4. Окно программы «Тест Черно-красная таблица» (Горбова-Шульте) и часть протокола прохождения теста

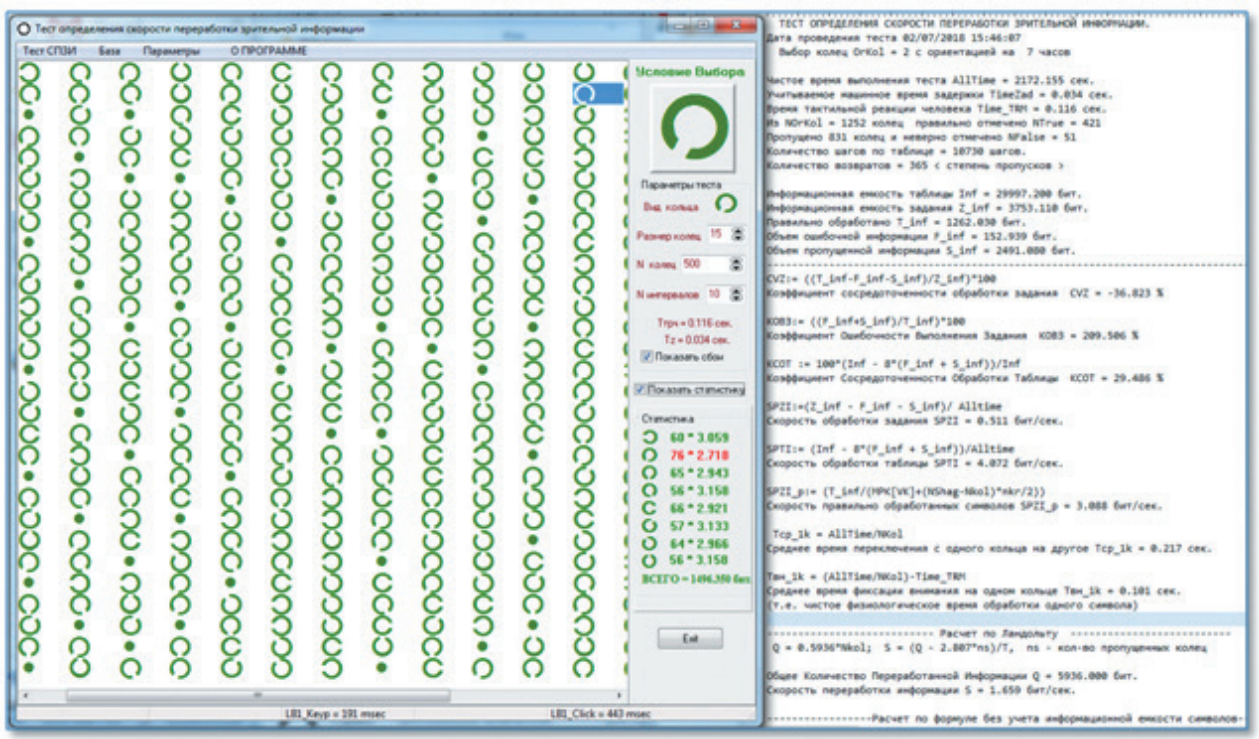

Рис. 5. Окно программы «Тест определения скорости переработки зрительной информации (ТСПЗИ)» и часть протокола прохождения теста
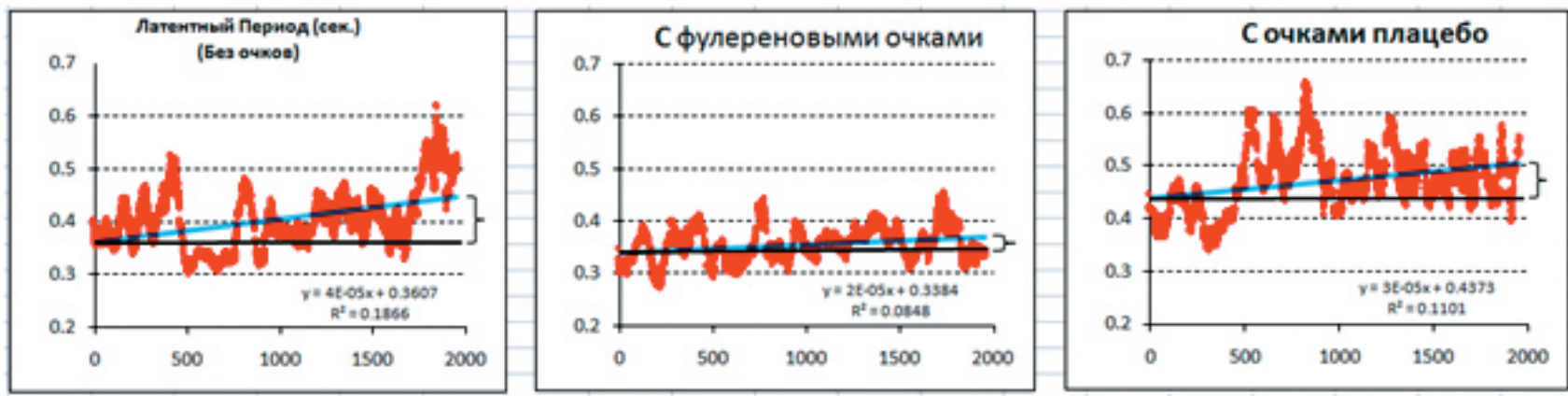

Рис. 6. Пример обработки результатов определения времени реакции на появление символа 
A

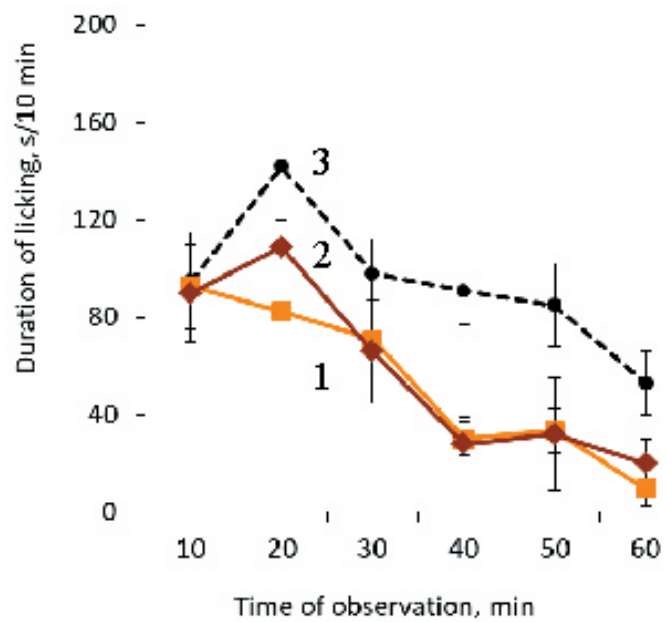

B

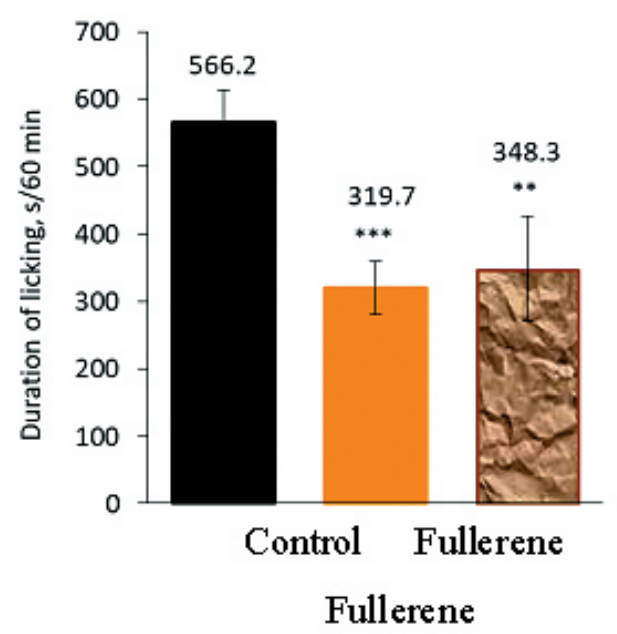

Рис. 7. Болевые реакции через 10 мин применения света аппарата БИОПТРОН, прошедшего через фуллереновый фильтр, на очаг боли (1) или на ТА Е-36 (2) по сравнению с контролем (3): А— динамика реакций в трех группах, В - общая продолжительность боли в течение 60 мин наблюдения. Столбики представляют среднее значение \pm квадратичная ошибка среднего. Цифры над столбиками - продолжительность болевых реакций в секундах. Достоверность различий с контролем: *** $-\mathrm{P}<0,001, * *-\mathrm{P}<0,05$

A

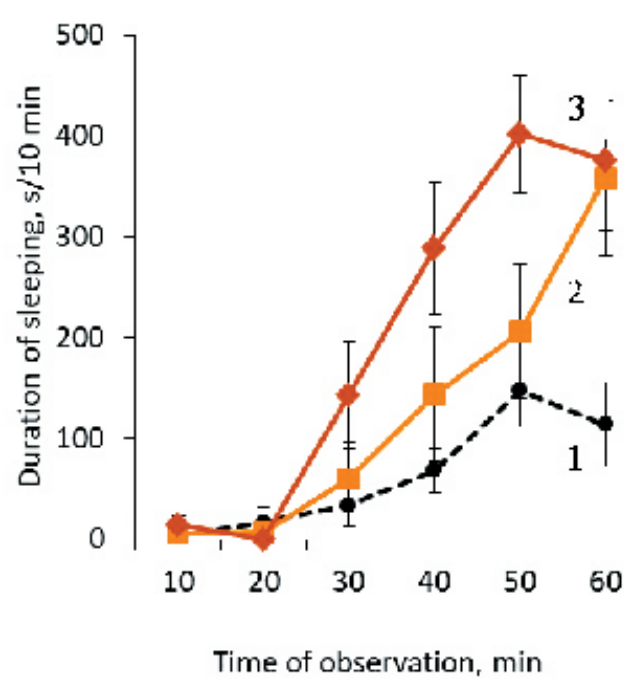

B

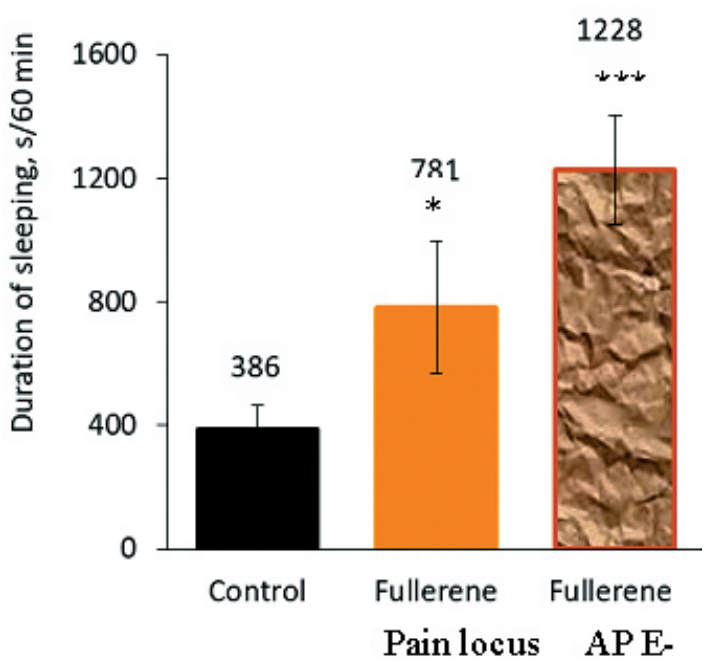

Рис. 8. Продолжительность сна после 10 мин применения света аппарата БИОПТРОН, проходящего через нанофотонный фуллереновый фильтр, на очаг боли (2) или на ТА Е-36 (3) по сравнению с контролем (1): А — динамика сна в трех группах; Б — общая продолжительность сна в течение 60 мин наблюдения. Столбцы представляют среднее значение \pm квадратичная ошибка среднего. Числа над столбиками — продолжительность сна в секундах. Достоверность различий с контролем: *** — $\mathrm{P}<0,001,{ }^{*}$ — $\mathrm{P}<0,5$ 

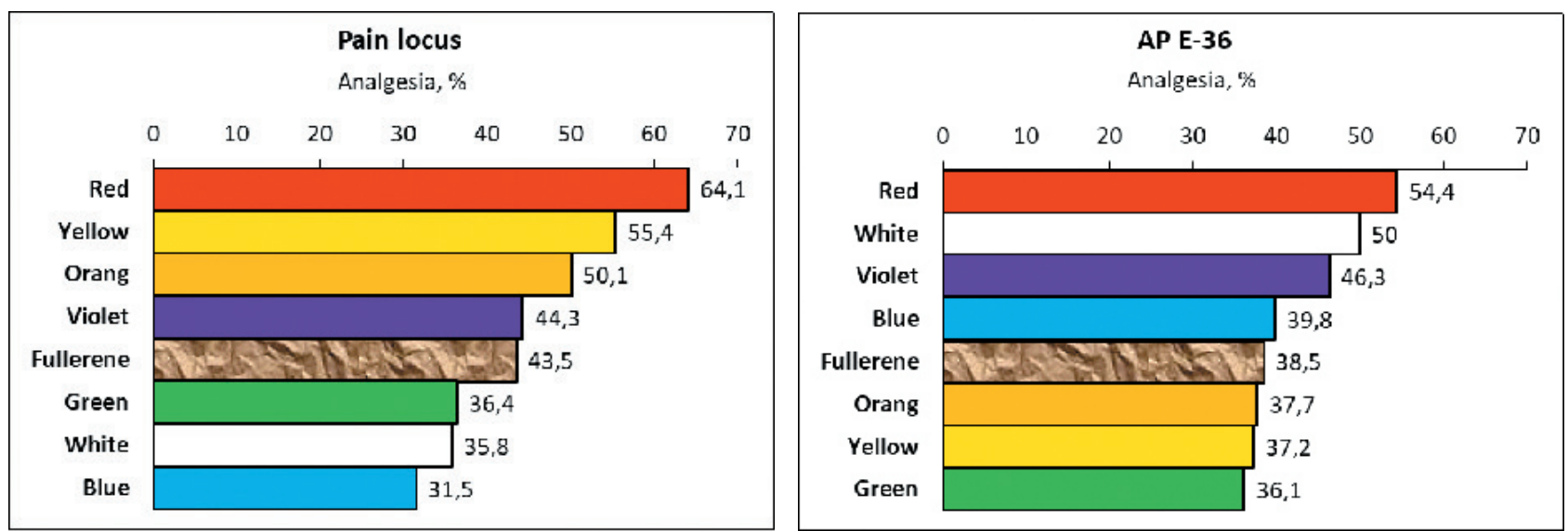

Рис. 9. Зависимость обезболивающего действия поляризованного света аппарата БИОПТРОН от волновых диапазонов различных фильтров: цифры над столбцами — означают величину анальгетической реакции в \%
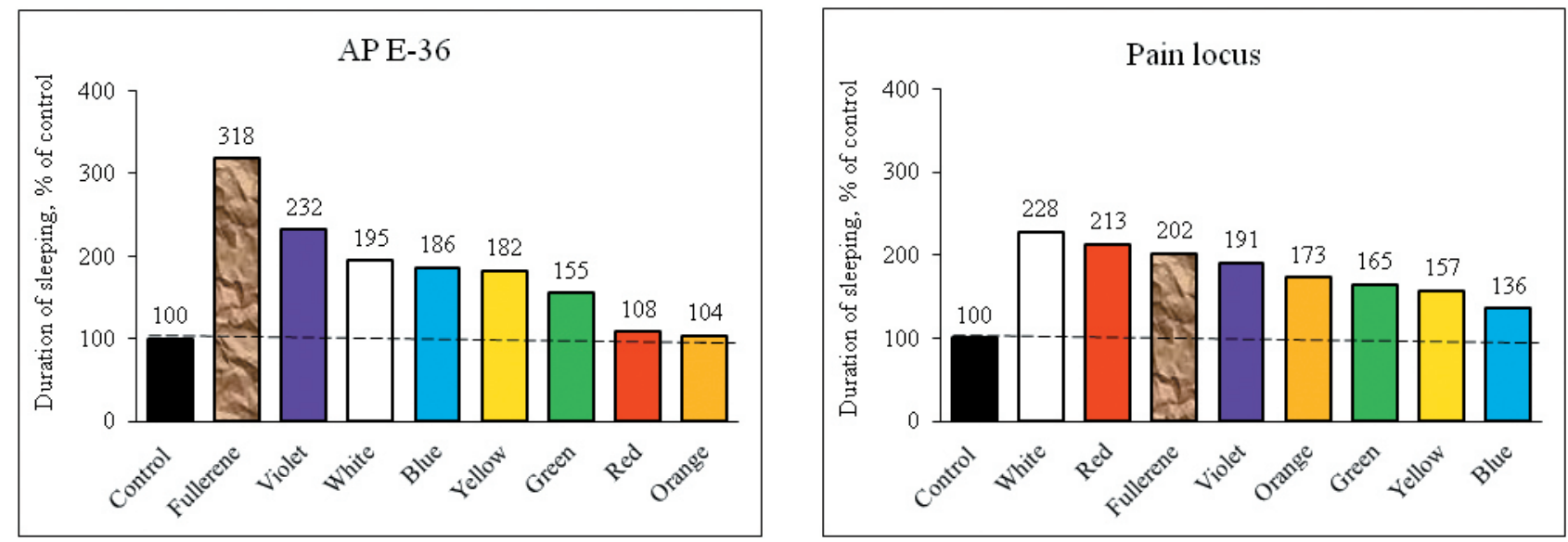

Рис. 10. Продолжительность сна в течение 60 мин наблюдения при боли, вызванной формалином, до и после действия поляризованным светом аппарата БИОПТРОН с различными фильтрами на ТА Е-36 или очаг боли: сон в контрольной группе принимается за 100 \%. Цифры над столбцами - средняя продолжительность сна в соответствующей группе в \% от контрольной. Экспериментальные группы расположены в порядке от максимальной до минимальной продолжительности сна

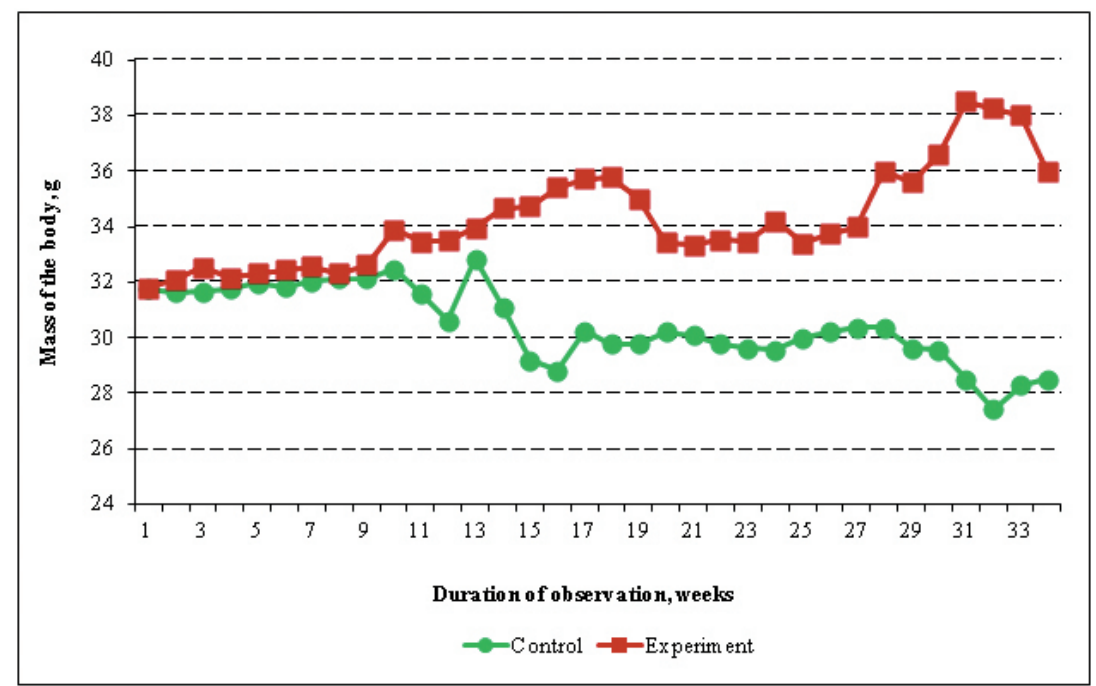

Рис. 11. Изменения массы тела у животных экспериментальной (фуллереновое освещение) и контрольной групп в течение 34 недель наблюдения: средние значения для каждой группы 


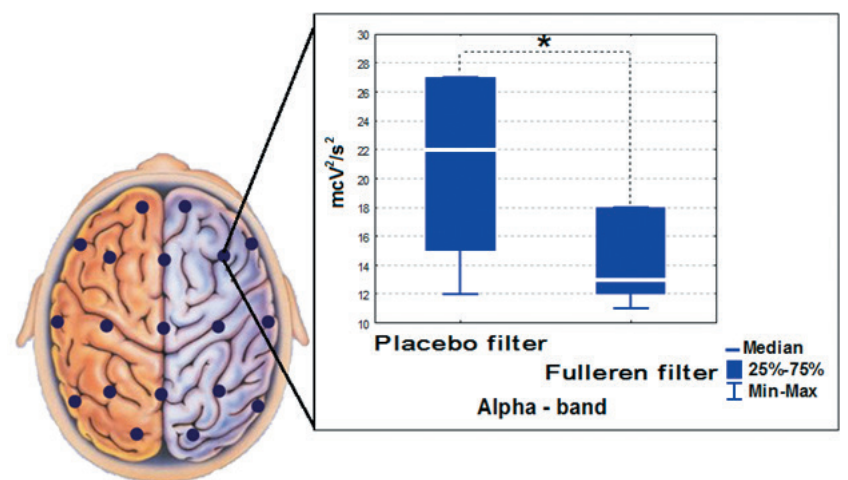

Рис. 12. Статистически значимые изменения активности мозга в правой лобной области коры человека под воздействием фуллеренового света (THE) в покое: по вертикали - спектральная плотность мощности; белая линия - медиана; прямоугольники - разброс данных (верхний и нижний квартили, 25 \% - 75 \%); вертикальные линии с разделителями - максимальные и минимальные значения показателей

Test duration

CNS age
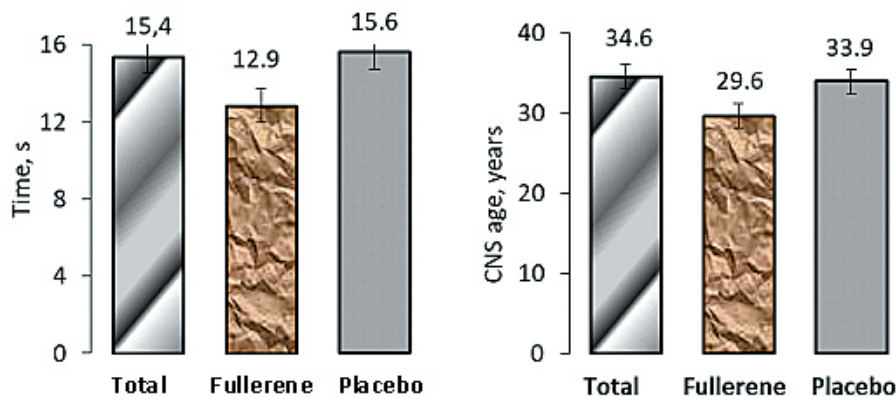

Рис. 13. Сравнение средних значений продолжительности выполнения чёрно-белого теста цифровой сортировки $(\mathrm{n}=15)$ и возраста участвующих в этом процессе структур ЦНС под действием фуллерен-модифицированного света в сравнении плацебо и интактом: указаны средние значения и стандартные погрешности среднего

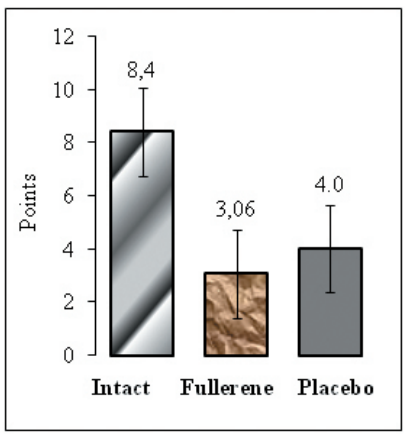

Рис. 14. Сравнение среднего количества ошибок допущенных при выполнении цветного цифрового теста (n=15), под действием фуллерен-модифицированного света в сравнении с плацебо и интактом: указаны средние значения и стандартные погрешности
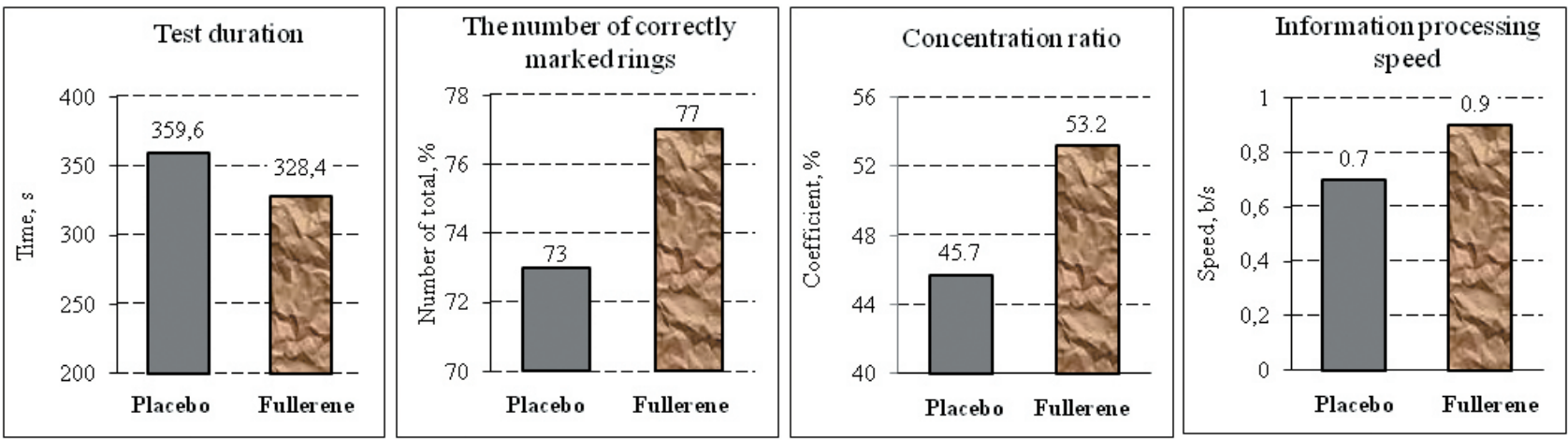

Рис. 15. Основные показатели ВНД при прямом корректурном тесте в очках-плацебо по сравнению с фуллереновыми очками ТНЕ (индивидуальные данные) 

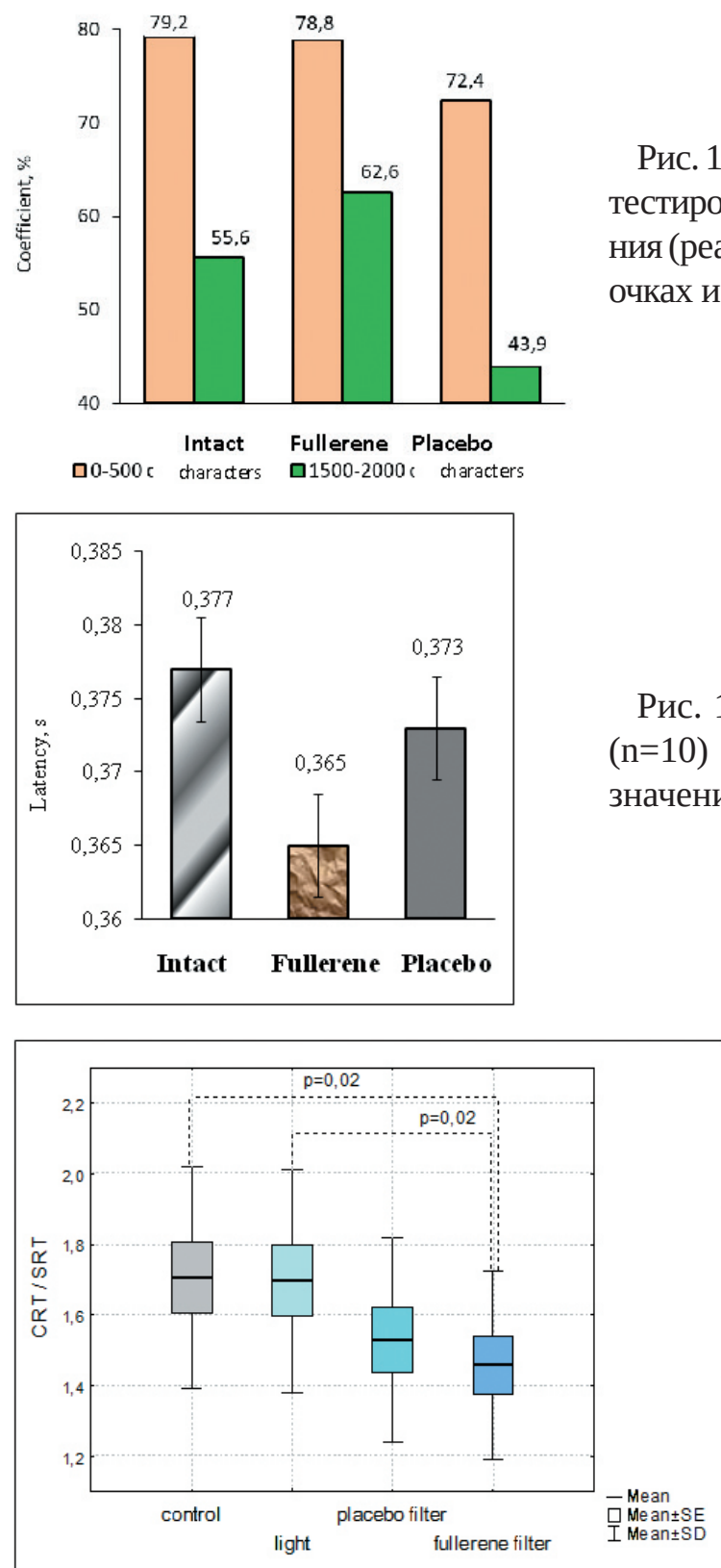

Рис. 16. Сравнение коэффициента сосредоточенности (\%) в начале тестирования (реакция на первые 500 символов) и в конце тестирования (реакция на последние 500 символов) без очков, в фуллереновых очках и в очках плацебо (индивидуальные данные)

Рис. 17. Латентный период реакции (с) на появление символа $(\mathrm{n}=10)$ в зависимости от зрительных условий: указаны средние значения \pm отклонение среднего

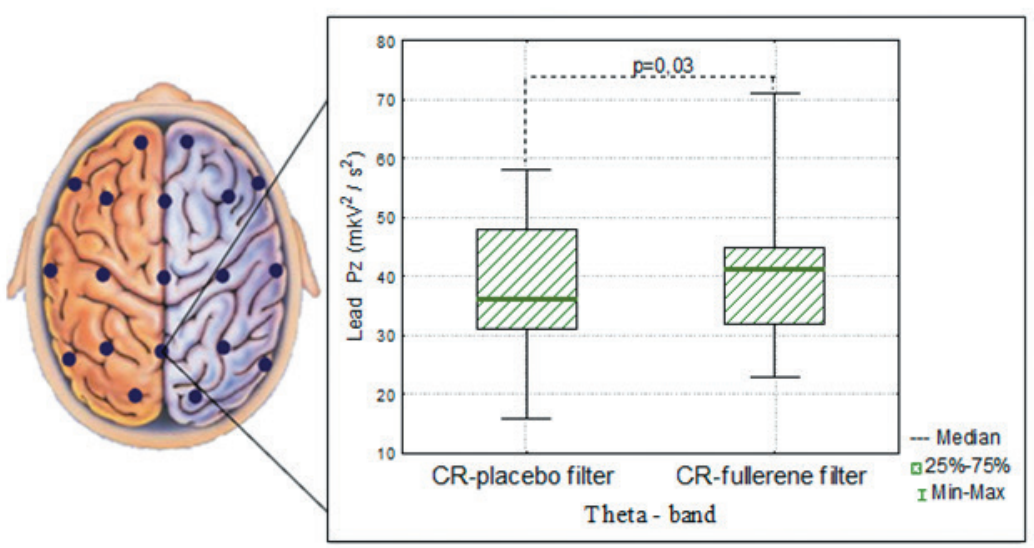

Рис. 19. Спектральная мощность ЭЭГ в тета-диапазоне при тестировании реакции выбора (CR) с использованием очков с плацебо фильтром и фуллереновым фильтром (fullerene filter) 
Эти данные свидетельствуют о том, что под влиянием света, модифицированного на наноуровне фуллереном, развивалось более созерцательное отношение к интроспективным эмоциональным переживаниям прошлых событий при активном вовлечении их в образное моделирование гипотетического будущего.

Исследования качества выполнения зрительномоторных тестов [33] выявили, что окулярное применение света, наномодифицированного фуллереном (очки), обусловило положительные изменения умственной работоспособности. Это подтверждалось сравнением результатов, полученных в очках Tesla HyperLight Eyewear в плацебо очках и в интактных условиях.

Выявлено, что средняя длительность выполнения чёрно-белого теста цифровой сортировки в плацебоочках (рис. 13) составляла 15,6 с (101,6 \% от контроля), а функциональный возраст структур ЦНС — 33,9 года (98.04 \% от контроля). В очках ТНЕ среднее время составляло 12,9 с (83,5 \% от контроля), а возраст ЦНС — 29,6 года (85,7 \% от контроля). Различия статистически достоверны. Это свидетельствовало о том, что эффект облегчения выполнения этого теста в очках ТНЕ является следствием преобразования света под действием примеси фуллерена в ПММА.

Умственная работоспособность в условиях постоянного выбора и переключения внимания (цветной красно-чёрный тест цифровой сортировки) у лиц, применявших очки THE была, наиболее высокой. Без очков возникало в среднем больше ошибок, чем в очках THE и очках плацебо. Сумма ошибок в баллах составляла 8, 4, 4.0 и 3.1 соответственно, что указывает на положительное влияние очков THE на внимательность (рис. 14). При этом длительность выполнения теста и скорость переключения внимания с одного символа на другой в очках ТНЕ существенно не отличалась от таковой в очках-плацебо. Вероятно, это обусловлено тем, что красные цифры из-за смещенного спектра выглядели менее контрастно.

Оценка индивидуальных данных выполнения простого корректурного теста показала, что чистая длительность выполнения теста составляла 328,400 с в очках ТНЕ против 359,574 с в очках-плацебо. Количество правильно отмеченных символов 77 и 73 \%, а скорость обработки задания 0,918 и 0,726 бит/с, соответственно. Другие показатели, характеризующие качество выполнения теста, в очках THE, также были лучше, чем в плацебоочках. Оцененные в баллах средние значения длительности прохождения теста, количества ошибок и выполнение теста в целом не отличались достоверно в случае тестирования без очков или в очках-плацебо. Следует отметить увеличение количества ошибок в очках-плацебо по сравнению с выполнением теста без очков. Оценка всех ошибок в баллах составляла 348,838 и 346,048 соответственно. Применение очков ТНЕ приводило к снижению количества ошибок при выполнении простого корректурного теста. Поскольку при обследовании испытуемых между ними всегда имеется значительный разброс показателей, различия особенно будут заметны при оценке индивидуальных данных (рис. 15).

Таким образом, во всех случаях свет, прошедший через очки ТНЕ облегчал прохождение тестов. Эти результаты позволяют заключить, что положительное влияние на выполнение зрительно-моторной задачи обусловлено именно наличием в очках примеси фуллерена, т. к. очки-плацебо не обладали таким эффектом.

Для оценки эффективности окулярного влияния наномодифицированного света при развитии утомления ЦНС сравнивали результаты, полученные при помощи 2-х специально разработанных тестов корректурной нагрузки, имитирующих длительную работу в социальных сетях. Простой корректурный тест на утомление требовал от испытуемого быстрой реакции на появление на экране компьютера символа (2000 колец Ландольта со случайным интервалом времени их возникновения). Корректурный тест на утомление с переключением внимания, требовал от испытуемого повышенного контроля ситуации дополнительно к скорости реакции. Необходимо было отмечать появляющиеся на экране кольца с заданной ориентацией разрыва («правильные», 10 \%). Наряду с этим на экране возникали «неправильные кольца» с другой ориентацией разрыва. Объем нагрузки был аналогичен.

Для простого корректурного теста установлено, что у обследуемых в очках ТНЕ увеличивалась скорость переработки информации. Уже в начале тестирования (при обработке первых 500 символов) скорость переработки информации в очках ТНЕ была выше $(1,05$ бит/с), чем без очков $(0,97)$ или в очках-плацебо $(0,98)$. Эта закономерность имела место и при обработке последних 500 символов $(0,95$, 0,87 и 0,68 бит/с).

Пребывание в очках THE также ослабляло утомляемость, которая неизбежно наблюдалась к концу тестирования. Если сравнить степень утомления у одного и того же испытуемого, то в фуллереновых очках она составляла $18 \%$. Тогда как без очков - 66\%, 
а в очках-плацебо - 29 \%. При применении очков ТНЕ повышался коэффициент сосредоточенности обработки задания. K концу выполнения задания без очков (интакт) у всех испытуемых имело место снижение коэффициента сосредоточенности. Однако при выполнении задания в очках ТНЕ (рис. 16) это снижение было наименышим (на 16,2\%) по сравнению с тестированием без очков (23,6 \%) или в очкахплацебо (28,5 \%). Скорость реакции на появление символа (латентный период) в очках ТНЕ была выше, чем без очков или в плацебо-очках. Средние по 10 испытуемым значения латентного периода реакции составляли: 0,38 $\pm 0,18$ с (без очков), 0,36 $\pm 0,15$ с (в

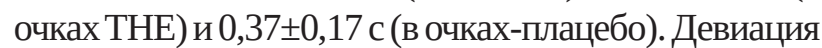
латентного периода реакции была наименьшей 0,26 $\pm 0,12$ с (в очках ТНЕ), против 0,29 $\pm 0,09$ с (без очков) и 0,28 $\pm 0,09$ с (в очках-плацебо).

Для корректурного теста с переключением внимания обнаружено, что реакция на любой из 2000 предъявляемых символов всегда была более быстрой, чем на «правильный» символ. Так, латентный период реакции на предъявление 2000 символов в среднем

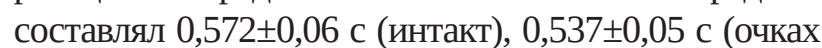

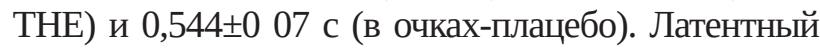
период реакции на «правильный» символ составляла 0,649 $\pm 0,09$ с (интакт), 0,623 $\pm 0,11$ с (в очках THE) и 0,636 $\pm 0,37$ с (в очках плацебо). Следует отметить, что в обоих случаях фуллереновые очки ТНЕ сокращали латентный период реакции по сравнению с тестированием без очков (рис. 17).

Установлено, что физиологическое время реакции (тест с переключением внимания) на появление одного символа без очков составляло 0,39 с, в очках THE - 0,36 с, а в плацебо-очках — 0,37 с. Таким образом, свет после фуллеренового нано-преобразования, укорачивал физиологическое время реакции. В этих же условиях сокращалось также общее время, затраченное на выполнение теста с переключением внимания. Без очков тест выполнялся в среднем за 280,3 с, а в очках THE — за 268,6 с.

Показателем качества выполнения теста является количество совершаемых ошибок. Оценка ошибок в баллах при выполнении теста с переключением внимания (n=9) показала следующие значения: без очков — 49,83 балла, в очках ТНЕ — 39,97 балла и в очках-плацебо — 46,11 балла. Таким образом, наименьшее количество ошибок испытуемые совершали при выполнении теста в очках THЕ. Следовательно, свет, прошедший через фуллереновый материал очков, способствует повышению внимательности и облегчает выполнение умственной нагрузки. То есть, оба теста длительной корректурной нагрузки показали, что свет после фуллереновой наномодификации способствует увеличению скорости переработки информации, повышает внимательность и ослабляет утомляемость.

Окулярное влияние света, нано-преобразованного фуллереном, оценивалось также по динамике простой сенсомоторной реакции, реакции выбора и их соотношения на фоне регистрации электрической активности мозга человека при экстремальных условиях освещения и неадекватной видимости объекта. Экспериментальная модель отражала стандартную ситуацию, в которую попадает водитель транспорта при ослепительно ярком освещении. Определялись латентные периоды простой сенсомоторной реакции и реакции выбора.

Выявлено снижение соотношения латентных периодов простой сенсомоторной реакции и реакции выбора при применении света, модифицированного фуллереном. Это свидетельствует об улучшении скорости межполушарных информационных процессов и повышении качества, эффективности принимаемых решений (рис. 18).

Найдено достоверное снижение активности нейронов в фронтальной и центральной зонах правого полушария в дельта- и бета2-диапазонах. Это указывает на снижение напряжения при идентификации стимула и ослабление активности процессов обработки изображений, т. е. развитие менее стрессорного состояния и облегчение принятия решений.

Доказано существенное увеличение активности нейронов, расположенных в задней сингулярной коре, в тета- и альфа-диапазонах (рис. 19). Это свидетельствует об улучшении координации зрительной информации, внимания, оценки положения своего тела, торможении нерелевантной информации и оптимизации процессов принятия решения. Следствием выявленных изменений стало увеличение скорости центральной обработки информации и смещение акцента с процессов обработки изображений на процессы принятия решений.

Описанные выше пилотные исследования показали, что под действием фуллереновых фильтров возникает две группы физиологических ответных реакций, которые целесообразно учитывать при клиническом и бытовом применении. Первая обусловлена созданием препятствия для прохождения «холодной» части видимого диапазона света. Вторая - последствия от превращения линейно 
поляризованного света во вращающийся в виде тороида Теслы «гиперполяризованный» квантовый поток. Теоретически за счет перестройки конфигурации получается более интенсивный (контрастный для восприятия) квантовый поток. В случае, если исходный свет находится в неполяризованном (рассеянном) состоянии, результирующий световой поток будет лишь частично трансформирован в гиперполяризованный за счет того, что часть квантов, направленных под разными углами к фильтру, будут от него отражаться. При этом на защитные (от синего излучения) свойства это не повлияет.

Дополнительные терапевтические возможности состоят в применении гиперполяризованного света в виде «усилителя» тех процессов, которые включаются в зависимости от зоны приложения света, экспозиции, мощности, периодичности и др. Однако, целесообразно учитывать наличие ослабления плотности мощности холодной части спектра. Это может создать предпочтение для фуллеренового фильтра при лечении патологий в области чувствительных к синей части спектра зон (глаз, открытых ран, ожоговых поверхностей и т. п.). Важен также результирующий антистрессорный эффект, который может быть получен при применении лечебных схем с использованием точек акупунктуры и других биологически активных зон. Имеет значение тот факт, что оранжево-красная часть света, проходящего через фуллереновый слой, не теряет свойств этого волнового диапазона. Комбинация его с красным светом, имеющим доказанное терапевтическое значение, создает предпосылки усиления конечного биологического результата.

Обращаем внимание на возникновение под действием фуллеренового света более заметного по сравнению с линейно поляризованным полихроматическим светом седативно-антистрессорного состояния. Поэтому для пациентов с симптомами стрессорности, перевозбуждения, судорожности, инсомнии будет оправданным переход в лечебных схемах на применение фуллереновых фильтров вместо стандартных полихроматических.

Окулярный путь светового воздействия (использование очков ТНЕ) выявил дополнительные возможности для коррекции психо-эмоционального равновесия и умственной работоспособности. В первую очередь применение фуллереновых очков будет полезным при работах с дисплеями, особенно при длительных экспозициях. Для водителей в условиях яркого дневного освещения есть шанс повышения безопасности движения и меньшей утомляемости, хотя при монотонной езде осторожность не должна ослабляться. Есть перспектива повышения качества умственной работы при подготовке текстов, поиске информации, одновременной работе в нескольких «окнах» социальных сетей и т. п., когда напряженная умственная деятельность ведет к утомлению и росту дефектов. В вечернее время ТНЕ, ослабляя световой фон, способствуют снятию дневного напряжения и улучшению качества сна. Во всех случаях будет обеспечено защитное действие фуллереновых фильтров за счет исключения влияния высокоэнергетических частей солнечного света - ультрафиолетового и синего.

Анализ источников литературы за счет того, что такое исследование является новым, проводить малоэффективно из-за отсутствия объекта сравнения. Тем не менее, авторы направляют читателей к списку литературы, в котором приведены первичные статьи [8, 23-26, 28-30, 33-34], имеющие конкретные аналитические фрагменты.

Выводы. 1. Экспериментально показано, что преобразованный фуллереном свет вызывает достоверные физиологическиеизменения, выявленные в антиноцицептивной сфере, ЦНС и ВНД.

2. На экспериментальной модели боли, вызванной подкожным введением формалина, установлено, что аппликация «углеродного» света на очаг боли/ воспаления или на точку акупунктуры вызывает достоверное ослабление болевой реакции (анальгетическое действие) и увеличение продолжительности сна (успокаивающее действие).

3. Сопоставление анальгетического действия отдельных основных монохроматических диапазонов показало достоверно высокую эффективность фуллеренового света. Седативный эффект, зарегистрированный при аппликации фуллеренового света на точку акупунктуры, достоверно превышал величины, полученные при иных световых диапазонах. Эти результаты подтверждают возможность как локального, так и удаленного (через точку акупунктуры) эффектов, получаемых бесконтактным и нефармакологическим путем.

4. Показано, что длительное (до 8 месяцев) освещение тела животного светом, прошедшим через фуллереновый фильтр, благоприятно действует на физиологические и функциональные показатели (динамика массы тела, сохранение двигательной активности, отсутствие негативных поведенческих изменений). Замедляется развитие некоторых признаков старения, таких как потеря массы тела и уменьшение подвижности. Достоверного влияния на 
продолжительность жизни животных не выявлено. Однако при этом длительность жизни отдельных особей значимыми превысила средние величины (до 14 мес). С учетом этих данных животные экспериментальной группы прожили суммарно 1452 дня, а контрольные животные - 1036 дней.

5. При окулярном действии фуллеренового света были получены достоверные отличия в дельта- и тета-диапазонах, которые указывали на изменения состояния дефолтных сетей. Выявлены сниженная ЭЭГ-активность в альфа-диапазоне в правой фронтальной зоне, повышенная ЭЭГ-активность в дельта-диапазоне в височной и задней височной зонах, повышенная ЭЭГ-активность в тета-диапазоне в задней сингулярной коре. Эти данные свидетельствуют о развитии более созерцательного отношения к интроспективным эмоциональным переживаниям прошлых событий при активном вовлечении их в образное моделирование гипотетического будущего.

6. Было установлено, что в естественных условиях применение фуллереновых очков облегчает выполнение зрительно-моторной задачи в чёрнобелом тесте цифровой сортировки на 16,5 \%. Это было эквивалентно снижению возраста структур ЦНС, принимавших участие в обработке цифровой информации, на 14,3 \%. Улучшалось качество работ, связанных с переключением внимания: по данным цветного цифрового теста длительность выполнения укорачивалась до 105,6 с против 118,0 с в контроле, среднее время переключения внимания с одного символа на другой - 1,85 с, против 2,03 с, а ошибок совершалось в 2,7 раза меньше.

7. При длительной корректурной нагрузке показано, что фуллереновый свет, способствует увеличению скорости переработки информации в зрительном анализаторе, повышает внимательность и ослабляет утомляемость. Коэффициент сосредоточенности снижался меныше (на 16,2 \%), чем в интактных (23, 6\%) или плацебо (28,5 \%) условиях. Латентный период реакции на появление символа был ниже, а его девиация была меньшей при сопоставлении с группами сравнения. Переключение внимания при нагрузке в 2000 символов всегда было более быстрым:

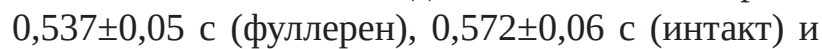

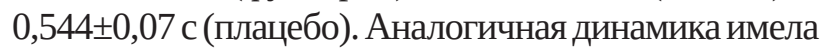
место для латентного периода реакции, ее физиологической длительности и времени, затраченного на выполнение теста с переключением внимания. Качество выполнения интенсивной умственной нагрузки при использовании фуллеренового света возрастало: количество ошибок составляло 39,97 балла, тогда, как в интактных условиях — 49,83, а плацебо - 46,11 баллов.

8. Окулярное влияние света, преобразованного фуллереном, на экспериментальной модели стандартной ситуации, в которую попадает водитель транспорта, позволила оценить динамику простой сенсомоторной реакции, реакции выбора и их соотношения на фоне регистрации электрической активности мозга человека. Выявлено снижение соотношения латентных периодов указанных реакции при применении света, модифицированного фуллереном. Это свидетельствует об улучшении скорости межполушарных информационных процессов и повышении качества и эффективности принимаемых решений. Доказано значимое увеличение активности нейронов, расположенных в задней сингулярной коре, в тета- и альфа-диапазонах. Это характеризует улучшение координации зрительной информации, внимания, оценки положения своего тела, торможение нерелевантной информации и оптимизацию процессов принятия решения. Следствием выявленных изменений стало увеличение скорости центральной обработки информации и смещение акцента с процессов обработки изображений на алгоритмы принятия решений. 


\section{Литература.}

1. Curl, R. F., Kroto, H., Smalley, R. E. (2017). The Nobel Prize in Chemistry 1996. Nobelprize. org. Nobel Media AB. Accessed August 31, 2017. http://www.nobelprize. org/nobel_prizes/chemistry/laureates/1996/.

2. Geim, A., Novoselov, K. (2017). The Nobel Prize in Physics 2010. Nobelprize. org. Nobel Media AB. Accessed September 1, 2017. http://www.nobelprize. org/nobel_prizes/physics/laureates/2010/.

3. Koruga, D., Hameroff, S., Loutfy, R., Withers, J., and Sundereshan, M. 1993. Fullerene C60: History, Physics, Nanobiology, Nanotechnology. Amsterdam: Elsevier (North Holland), 1-381.

4. Koruga, D., Simic-Krstic, J., Trifunovic, M., Jankovic, S., Hameroff, S., Withers, J., and Loutfy, R. (1993). Imaging Fullerene C60 with Atomic Resolution Using Scanning Tunneling Microscope. Fullerene Science and Technology, 1 (1), 93-100.

5. Koruga, D., Matija, L., Misic, N., and Rakin, P. (1996). Fullerene C60: Properties and Possible Applications. Materials Science Forum, 214, 49-56.

6. Morton, J. J. L., Tyryshkin, A. M., Ardavan, A., Benjamin, S. C., Porfyrakis, K., Lyon, S. and Briggs, G. A. D. (2006). Bang-bang Control of Fullerene Cubits Using Ultrafast Phase Gates. Nature Physics., 2, 40-43.

7. Koruga, D. (2016). Nanophotonic filter based on C60 for hyperpolarized light. Int. and European Pat. App. PCT/EP2016/063174. Applicant: Field point, ZEPTER GROUP filed June 09, 2016 and issued October 28, 2016.

8. Koruga, D. (2017). Hyperpolarized Light: Fundamentals of Nano Medical Photonics. Belgrade: Zepter World Book, 1-306.

9. Baranova, E. (2010). Graphene is Characterized by a Giant Faraday Rotation. Sci-Lib. Accessed November 18, 2010. http://sci-lib.com/article912.html.

10. Litchinitser, N. (2012). Structured Light Meets Structured Matter. Science, 337 (6098), 1054-5.

11. Gulyar, S. A., Limansky, Yu. P., Tamarova, Z. A. (2004). Pain and color. Kyiv-Donetzk: Biosvet, 1-122.

12. Tamarova, Z. A., Limansky, Yu. P., Gulyar, S. A. (2009). Antinociceptive Effects of Color Polarized Light in Animal Formalin Test Model. Fiziolohichnyi Zhurnal, 55 (3), 81-93.

13. Gulyar, S. A., Kosakovskyi, A. L., eds. (2011). BioptronPILER-Light Application in Medicine (2nd ed.). Kyiv: Bogomoletz Institute of Physiology at the National Academy of Sciences of Ukraine, 1-256.

14. Limansky, Yu. P., Gulyar, S. A., Tamarova, Z. A., Bydkov, E. G. (2000). Investigation of Analgesic Action of Polarized Light. Fiziologichnyi Zhurnal, 46 (6), 105-111.

15. Zagryadskiy, V. P, Sulymo-Samuylo, Z. K. (1976). Research Methods in the Physiology of Labor. Leningrad: Nauka, 93.

16. Rymsky, S., Rymskaya, R. (1995). Almanac of Psychological Tests. Moscow: KSP+.
17. Burlachuk, A. F., Morozov, S. M. (1989). DictionaryReference Book in Psychological Diagnostics. Kyiv: Naukova dumka, 61-62.

18. State Standard USSR 12.4.061-79. (1981). Method for determining human performance in personal protective equipment. Occupational Safety Standards System, ch. 2, Moskow, 121-133.

19. Polyschuk, I. A., Vydrenko, A. E. (1979). Atlas for an Experimental Study of Abnormalities in Human Mental Activity. Kyiv: Zdorovia.

20. Myronova, E. E. (2006). Collection of Psychological Tests. Part II. Allowance. Minsk: ENVILA Women's Institute, 1-146.

21. Genkyn, A. A., Medvedev, V. I, Shek, M. P. (1963). Some principles of the construction of correction tables for determining the speed of information processing. Questions of psychology, 1, 104- 107.

22. Rebrova O. (2002). Statistical analysis of medical data. Application of software package Statistica. Moskow: MediaSfera, 1-312.

23. Gulyar, S. A., Tamarova, Z. A. (2017). Anti-Pain and Sedative Action of Polychromatic Polarized Light which Passed Through Nanomodification by Carbon Nanofilters. Proc. of the XLVII International Scientific and Practical Conference Proceedings of Academic Science, Oct. 12-14, 2017, Kyiv, 95-97.

24. Gulyar, S. A., Tamarova, Z. A. (2017). Modification of Polychromatic Linear Polarized Light by Nanophotonic Fullerene and Graphene Filter Creates a New Therapeutic Opportunities. Journal of US-China Medical Science, 14 (5), 173-91.

25. Gulyar, S. A. (2018). From Polarization to Hyperpolarization: New Aspects of Light Therapy. Proceeding of the XLIX International Scientific and Practical Conference Proceedings of Academic Science and 22nd Gamaleya's Readings. Oct. 3-7, 2018. Hajduszoboszlo, 157-162.

26. Gulyar, S. A., Tamarova, Z. A. (2018). Influence of Many-Month Exposure to Light with Shifted Wave Range and Partial Fullerene Hyperpolarization on the State of Elderly Mice. Journal of US-China Medical Science, 15 (1), 16-25.

27. Raichle, M. E., MacLeod, A. M., Snyder, A. Z., Powers, W. J., Gusnard, D. A., and Shulman, G. L. (2001). A Default Mode of Brain Function. Proc. of the National Academy of Sciences. Oct. 12-14, 2017, Kyiv, 98 (11), 676-82.

28. Filimonova, N. B., Makarchuk, N. E., Gulyar, S. A. (2017). Influence of Short-term Ocular Exposition of Fullerene Light on the Activity of Default Chains of the Human Brain. Proc. of the XLVII International Scientific and Practical Conference, 118-120.

29. Filimonova, N. B., Makarchuk, N. E., Gulyar, S. A., Kryvdiuk, Yu. N. (2018). Influence of Light, Modified by Fulleren, on the Speed of the Brain Interhemispheric Information Processes on the Background of Changed Brightness of the Light Stimulus. Proc. of the XLIX 
International Scientific and Practical Conference and 22nd Gamaleya’s Readings, Oct. 3-7, 2018. Hajduszoboszlo, 163-167.

30. Gulyar, S. A., Filimonova, N. B., Makarchuk, M. Yu., and Kryvdiuk, Y. N. (2019). Ocular Influence of Nano-Modified Fullerene Light: 1. Activity of Default Networks of the Human Brain. Journal of US-China Medical Science, 16 (2), 1-15.

31. Klimesch, W. (1999). EEG Alpha and Theta Oscillations Reflect Cognitive and Memory Performance. Brain Research Reviews, 29 (2), 169-195.

32. Bernat, E., Lindsay, D., Holroyd, B., Gehring, W. (2008). Separating Cognitive Processes with Principal Components Analysis of EEG Time-frequency Distributions. Advanced Signal Processing Algorithms, Architectures, and Implementations XVIII International Society for Optics and Photonics, 326-33.

33. Gulyar, S. A., Tamarova, Z. A., Kirilenko, E. K. (2018). Peculiarities of Human Mental Workability Under the Oculary Influence of Light Transformed by Nanophotonic Filter. Proc. of the XLIX International Scientific and Practical Conference and 22nd Gamaleya's Readings, Oct. 3-7, 2018. Hajduszoboszlo, 59-62.

34. Gulyar, S. A. (2018). Accents of the Human Body Electromagnetic Balance Regulation System. Photobiology and Photomedicine, 24, 52-68.

\section{References.}

1. Curl, R. F., Kroto, H., Smalley, R. E. (2017). The Nobel Prize in Chemistry 1996. Nobelprize. org. Nobel Media AB. Accessed August 31, 2017. http://www.nobelprize. org/nobel_prizes/chemistry/laureates/1996/.

2. Geim, A., Novoselov, K. (2017). The Nobel Prize in Physics 2010. Nobelprize. org. Nobel Media AB. Accessed September 1, 2017. http://www.nobelprize. org/nobel_prizes/physics/laureates/2010/.

3. Koruga, D., Hameroff, S., Loutfy, R., Withers, J., and Sundereshan, M. 1993. Fullerene C60: History, Physics, Nanobiology, Nanotechnology. Amsterdam: Elsevier (North Holland), 1-381.

4. Koruga, D., Simic-Krstic, J., Trifunovic, M., Jankovic, S., Hameroff, S., Withers, J., and Loutfy, R. (1993). Imaging Fullerene C60 with Atomic Resolution Using Scanning Tunneling Microscope. Fullerene Science and Technology, 1 (1), 93-100.

5. Koruga, D., Matija, L., Misic, N., and Rakin, P. (1996). Fullerene C60: Properties and Possible Applications. Materials Science Forum, 214, 49-56.

6. Morton, J. J. L., Tyryshkin, A. M., Ardavan, A., Benjamin, S. C., Porfyrakis, K., Lyon, S. and Briggs, G. A. D. (2006). Bang-bang Control of Fullerene Cubits Using Ultrafast Phase Gates. Nature Physics., 2, 40-43.

7. Koruga, D. (2016). Nanophotonic filter based on C60 for hyperpolarized light. Int. and European Pat. App. PCT/EP2016/063174. Applicant: Field point, ZEPTER GROUP filed June 09, 2016 and issued October 28, 2016.
8. Koruga, D. (2017). Hyperpolarized Light: Fundamentals of Nano Medical Photonics. Belgrade: Zepter World Book, 1-306. [In Serbian and in English].

9. Baranova, E. (2010). Graphene is Characterized by a Giant Faraday Rotation. Sci-Lib. Accessed November 18, 2010. http://sci-lib.com/article912.html. [In Russian].

10. Litchinitser, N. (2012). Structured Light Meets Structured Matter. Science, 337 (6098), 1054-5.

11. Gulyar, S. A., Limansky, Yu. P., Tamarova, Z. A. (2004). Pain and color. Kyiv-Donetzk: Biosvet, 1-122.

12. Tamarova, Z. A., Limansky, Yu. P., Gulyar, S. A. (2009). Antinociceptive Effects of Color Polarized Light in Animal Formalin Test Model. Fiziolohichnyi Zhurnal, 55 (3), 81-93.

13. Gulyar, S. A., Kosakovskyi, A. L., eds. (2011). BioptronPILER-Light Application in Medicine (2nd ed.). Kyiv: Bogomoletz Institute of Physiology at the National Academy of Sciences of Ukraine, 1-256. [In Russian].

14. Limansky, Yu. P., Gulyar, S. A., Tamarova, Z. A., Bydkov, E. G. (2000). Investigation of Analgesic Action of Polarized Light. Fiziologichnyi Zhurnal, 46 (6), 105111. [In Russian].

15. Zagryadskiy, V. P, Sulymo-Samuylo, Z. K. (1976). Research Methods in the Physiology of Labor. Leningrad: Nauka, 93. [In Russian].

16. Rymsky, S., Rymskaya, R. (1995). Almanac of Psychological Tests. Moscow: KSP+. [In Russian].

17. Burlachuk, A. F., Morozov, S. M. (1989). DictionaryReference Book in Psychological Diagnostics. Kyiv: Naukova dumka, 61-62. [In Russian].

18. State Standard USSR 12.4.061-79. (1981). Method for determining human performance in personal protective equipment. Occupational Safety Standards System, ch. 2, Moskow, 121-133. [In Russian].

19. Polyschuk, I. A., Vydrenko, A. E. (1979). Atlas for an Experimental Study of Abnormalities in Human Mental Activity. Kyiv: Zdorovia. [In Russian].

20. Myronova, E. E. (2006). Collection of Psychological Tests. Part II. Allowance. Minsk: ENVILA Women’s Institute, 1-146. [In Russian].

21. Genkyn, A. A., Medvedev, V. I, Shek, M. P. (1963). Some principles of the construction of correction tables for determining the speed of information processing. Questions of psychology, 1, 104 - 107. [In Russian].

22. Rebrova O. (2002). Statistical analysis of medical data. Application of software package Statistica. Moskow: MediaSfera, 1-312. [In Russian].

23. Gulyar, S. A., Tamarova, Z. A. (2017). Anti-Pain and Sedative Action of Polychromatic Polarized Light which Passed Through Nanomodification by Carbon Nanofilters. Proc. of the XLVII International Scientific and Practical Conference Proceedings of Academic Science, Oct. 12-14, 2017, Kyiv, 95-97.

24. Gulyar, S. A., Tamarova, Z. A. (2017). Modification of Polychromatic Linear Polarized Light by Nanophotonic 
Fullerene and Graphene Filter Creates a New Therapeutic Opportunities. Journal of US-China Medical Science, 14 (5), 173-91.

25. Gulyar, S. A. (2018). From Polarization to Hyperpolarization: New Aspects of Light Therapy. Proceeding of the XLIX International Scientific and Practical Conference Proceedings of Academic Science and 22nd Gamaleya’s Readings. Oct. 3-7, 2018. Hajduszoboszlo, 157-162.

26. Gulyar, S. A., Tamarova, Z. A. (2018). Influence of Many-Month Exposure to Light with Shifted Wave Range and Partial Fullerene Hyperpolarization on the State of Elderly Mice. Journal of US-China Medical Science, 15 (1), 16-25.

27. Raichle, M. E., MacLeod, A. M., Snyder, A. Z., Powers, W. J., Gusnard, D. A., and Shulman, G. L. (2001). A Default Mode of Brain Function. Proc. of the National Academy of Sciences. Oct. 12-14, 2017, Kyiv, 98 (11), 676-82.

28. Filimonova, N. B., Makarchuk, N. E., Gulyar, S. A. (2017). Influence of Short-term Ocular Exposition of Fullerene Light on the Activity of Default Chains of the Human Brain. Proc. of the XLVII International Scientific and Practical Conference, 118-120.

29. Filimonova, N. B., Makarchuk, N. E., Gulyar, S. A., Kryvdiuk, Yu. N. (2018). Influence of Light, Modified by Fulleren, on the Speed of the Brain Interhemispheric Information Processes on the Background of Changed
Brightness of the Light Stimulus. Proc. of the XLIX International Scientific and Practical Conference and 22nd Gamaleya's Readings, Oct. 3-7, 2018. Hajduszoboszlo, 163-167.

30. Gulyar, S. A., Filimonova, N. B., Makarchuk, M. Yu., and Kryvdiuk, Y. N. (2019). Ocular Influence of Nano-Modified Fullerene Light: 1. Activity of Default Networks of the Human Brain. Journal of US-China Medical Science, 16 (2), 1-15.

31. Klimesch, W. (1999). EEG Alpha and Theta Oscillations Reflect Cognitive and Memory Performance. Brain Research Reviews, 29 (2), 169-195.

32. Bernat, E., Lindsay, D., Holroyd, B., Gehring, W. (2008). Separating Cognitive Processes with Principal Components Analysis of EEG Timefrequency Distributions. Advanced Signal Processing Algorithms, Architectures, and Implementations XVIII International Society for Optics and Photonics, 326-33.

33. Gulyar, S. A., Tamarova, Z. A., Kirilenko, E. K. (2018). Peculiarities of Human Mental Workability Under the Oculary Influence of Light Transformed by Nanophotonic Filter. Proc. of the XLIX International Scientific and Practical Conference and 22nd Gamaleya's Readings, Oct. 3-7, 2018. Hajduszoboszlo, 59-62.

34. Gulyar, S. A. (2018). Accents of the Human Body Electromagnetic Balance Regulation System. Photobiology and Photomedicine, 24, 52-68. 